\title{
多巴胺辅助牙本质沉积羟基磷灰石研究
}

\author{
许金妹，刘新玲，高彦峰 \\ (上海大学 材料科学与工程学院, 上海 200444)
}

\begin{abstract}
摘 要: 本研究报道了一种新颖的、模拟口腔环境的仿生修复牙齿的方法。利用具有良好生物相容性的多巴胺在牛 牙牙本质表面自聚合形成聚多巴胺层(PDA)使牙本质表面功能化, 进而诱导羟基磷灰石从模拟唾液中向牙本质表 面沉积。XPS、XRD、SEM 和 EDS 表征分析表明：聚多巴胺层的涂覆有利于牙本质表面羟基磷灰石晶体的结晶生 长, 可提高羟基磷灰石再矿化层与牙本质表面的结合力; 多巴胺溶液浓度为 $2 \mathrm{mg} / \mathrm{mL}$ 时, 牙本质表面聚多巴胺层的 沉积效果最佳。
\end{abstract}

关 键 词: 仿生材料; 多巴胺自聚合; 牙齿再矿化; 结晶生长

中图分类号: R314 文献标识码: A

\section{Effect of Dopamine on Hydroxyapatite Deposition for Dental Restoration}

\author{
XU Jin-Mei, LIU Xin-Ling, GAO Yan-Feng \\ (School of Materials Science and Engineering, Shanghai University, Shanghai 200444, China)
}

\begin{abstract}
A novel and biomimetic restoration method for teeth by soaking in a simulated oral environment was reported. A biocompatible polydopamine (PDA) layer was deposited on the surface of dentin via dopamine polymerization to functionalize the surface to induce deposition of hydroxyapatite crystals. The samples were characterized by means of XPS, XRD, SEM and EDS. Results show that the coating of polydopamine layer induces the growth of hydroxyl apatite crystal on dentin surface, and improve the binding force between the hydroxyl apatite layer and the dentin surface. The optimized concentration of dopamine solution is $2 \mathrm{mg} / \mathrm{mL}$ for the uniform deposition of PDA layer on the dentin surface.
\end{abstract}

Key words: biomimetic materials; polymerization of dopamine; mineralization of teeth; crystal growth

龊病是牙齿硬组织结构逐渐被破坏的一种慢性疾 病 $^{[1]}$, 一直以来影响着人们的生活, 危害着人类的口腔 健康。龊齿的发生从牙釉质的脱矿开始, 因此脱矿牙釉 质的修复研究对于䠗齿的预防和治疗具有重要意义 ${ }^{[2-4]}$ 。 虽然现在的口腔材料本身对于修复龊洞或者牙齿缺损 较为有效, 但是无法达到防止嚙齿再次发生 ${ }^{[5]}$ 的目的。 理想的口腔修复材料在组成、结构和性质等方面应该 与牙齿组织相似, 所以与牙釉质组成类似的羟基磷灰 石材料即为牙齿修复的理想材料 ${ }^{[6]}$ 。

目前, 关于这方面的研究主要集中在牙釉质的 模型矿物一一羟基磷灰石的合成、应用以及生物矿
化过程的模拟、机理等方面。Yamagishi 等 ${ }^{[7]}$ 开发了 一种以氟磷酸钙为主的胶体，可以在酸性母液的配 合下直接用于牙釉质缺损的修复。Mansfield 等 $^{[8]}$ 模 拟生物矿化过程中蛋白质对无机矿物的组装行为, 获得了具有与牙釉质结构类似的自组装体。 Clarkson 等 ${ }^{[9]}$ 利用水热方法合成了具有柱状结构的 纳米羟基磷灰石晶体, 在合成过程中这些晶体进行 自组装形成了具有类牙釉的组装结构。但是, 这些 方法操作条件较为苛刻, 实用性差。

当牙釉质全部溶蚀后, 便会暴露牙本质, 引起 牙齿过敏。因此, 在牙本质表面重新形成一层保护层

收稿日期: 2015-06-02; 收到修改稿日期：2015-07-18

基金项目: 国家杰出青年科学基金(51325203) National Science Fund for Distinguished Young Scholars(51325203)

作者简介：许金妹(1991-)，女，硕士研究生. E-mail: xujinmei1222@126.com

通讯作者：高彦峰，研究员. E-mail: yfgao@shu.edu.cn 
也是解决牙齿过敏的方法之一。本研究利用仿生矿 化的方法来达到牙齿修复的目的, 主要包括两个方 面: 第一是在受损牙本质表面实现多巴胺的氧化自 聚合 ${ }^{[10-12]}$, 构建牙本质再矿化的二级反应“桥梁”; 第 二是通过聚多巴胺层的功能化作用, 在模拟唾液中 沉积出羟基磷灰石以实现牙本质的再矿化。该方法不 受基材尺寸、形状的限制, 而且聚多巴胺具有良好的 生物相容性, 为仿生牙齿修复材料的研究及脱矿牙本 质的自愈性修复提供了新的理论和技术基础。

\section{1 实验方法}

\section{1 牙片表面聚多巴胺涂层的制备}

实验选取新拔取的黄牛牙, 用 $3 \%$ 次氯酸钠处理 以去除菌斑, 并用磷酸盐缓冲液 $(135 \mathrm{mmol} / \mathrm{L} \mathrm{NaCl}$ 、

$2.7 \mathrm{mmol} / \mathrm{L} \mathrm{KCl} 、 1.5 \mathrm{mmol} / \mathrm{LH}_{2} \mathrm{PO}_{4} 、 8 \mathrm{mmol} / \mathrm{L}$ $\mathrm{K}_{2} \mathrm{HPO}_{4}, \mathrm{pH}=7.2$ )漂洗。用低速 STX-202A 型金刚石 线切割机将牙齿在有水冷却的情况下, 垂直于牙齿 的纵轴将牙冠切成厚约 $0.5 \sim 1 \mathrm{~mm}$ 、直径约 $8 \sim 10 \mathrm{~mm}$ 的平片。用 600 3000 目砂纸将牙片表面打磨光滑, 之后牙片样本依次用去污剂、丙酮、乙醇、去离子 水超声清洗, 室温干燥, 放在聚乙烯管里备用。用 $37 \%$ 磷酸酸蚀准备好的牛牙片 $2 \mathrm{~min}$, 再用足够的去 离子水漂洗。选取其中一牙片作为空白对照组, 编 号为 $\mathrm{a}$ 组, 剩余牙片无需干燥, 直接分别浸没于新 鲜配制的 1、2、3 mg/mL 多巴胺(Sigma-Aldrich, St. Louis, MO)溶液(其 $\mathrm{pH}$ 由 $10 \mathrm{mmol} / \mathrm{L}$ tris- $\mathrm{HCl}$ 缓冲液 和 $0.1 \mathrm{~mol} / \mathrm{L} \mathrm{HCl}$ 调至 8.5)中室温避光存放 $24 \mathrm{~h}$ 后, 取出样品用去离子水超声清洗 $10 \mathrm{~min} \times 3$ 次, 以去除 没有结合的多巴胺, 室温干燥可得到涂覆有聚多巴
胺涂层的牙片，分别编号为 $b 、 c 、 d 。$

\section{2 涂覆有聚多巴胺(PDA)涂层的牙体组织的} 再矿化

参照 Fan 等 ${ }^{[13]}$ 描述的操作步骤配制矿化液。将 步骤 1.1 中处理后的涂覆有聚多巴胺涂层牙片(实验 组 b、c、d) 和空白牙片(对照组 a)分别放置在含 $50 \mathrm{~m}$ $\mathrm{L}$ 矿化液的密封烧杯的底部 ${ }^{[14]}$ 。然后将烧杯放在 $37^{\circ} \mathrm{C}$ 的恒温摇床上以 $100 \mathrm{r} / \mathrm{min}$ 速度持续震荡。每 天更换一次矿化液, $7 \mathrm{~d}$ 后取出样品, 用流动的去离 子水漂洗，再用 $50 \% 、 70 \% 、 80 \% 、 90 \% 、 95 \% 、 100 \%$ 乙醇进行上行梯度脱水, 用每个浓度的乙醇脱水 2 次, 每次 $10 \mathrm{~min}$, 将脱水后的样本干燥后存于冰箱。

\section{3 测试表征}

采用型号为 ESCALAB 250Xi 的 X 射线光电子能 谱仪测试牙本质表面聚多巴胺层涂覆状况; 利用 JSM-6700F 扫描电子显微镜(SEM)对牙本质表面聚多 巴胺层以及再矿化层的表面形貌进行观察，并通过 SEM 自带的 EDS 来表征所选区域内的元素含量; 采用 $18 \mathrm{KWD} / \mathrm{MAX} 2500 \mathrm{~V}+\mathrm{PC}$ 型 X 射线衍射仪测定牙本质 表面矿化沉积物的 XRD 图谱, 测试条件为: $\mathrm{CuK \alpha}$, 最大 额定输出为 $18 \mathrm{~kW}$, 测试时的管压与管流分别为 $40 \mathrm{kV}$ 、 $200 \mathrm{~mA}$, 扫描速度为 $8^{\circ} / \mathrm{min}$, 扫描范围 $2 \theta=20^{\circ} \sim 60^{\circ}$ 。

\section{2 结果与讨论}

\section{1 多巴胺在牙本质表面聚合沉积状况的 SEM 形貌分析}

在扫描电镜下，可以观察到这些天然牙本质典 型的孔状结构(图 1(a), (b))。在对照组实验中, 当牙片
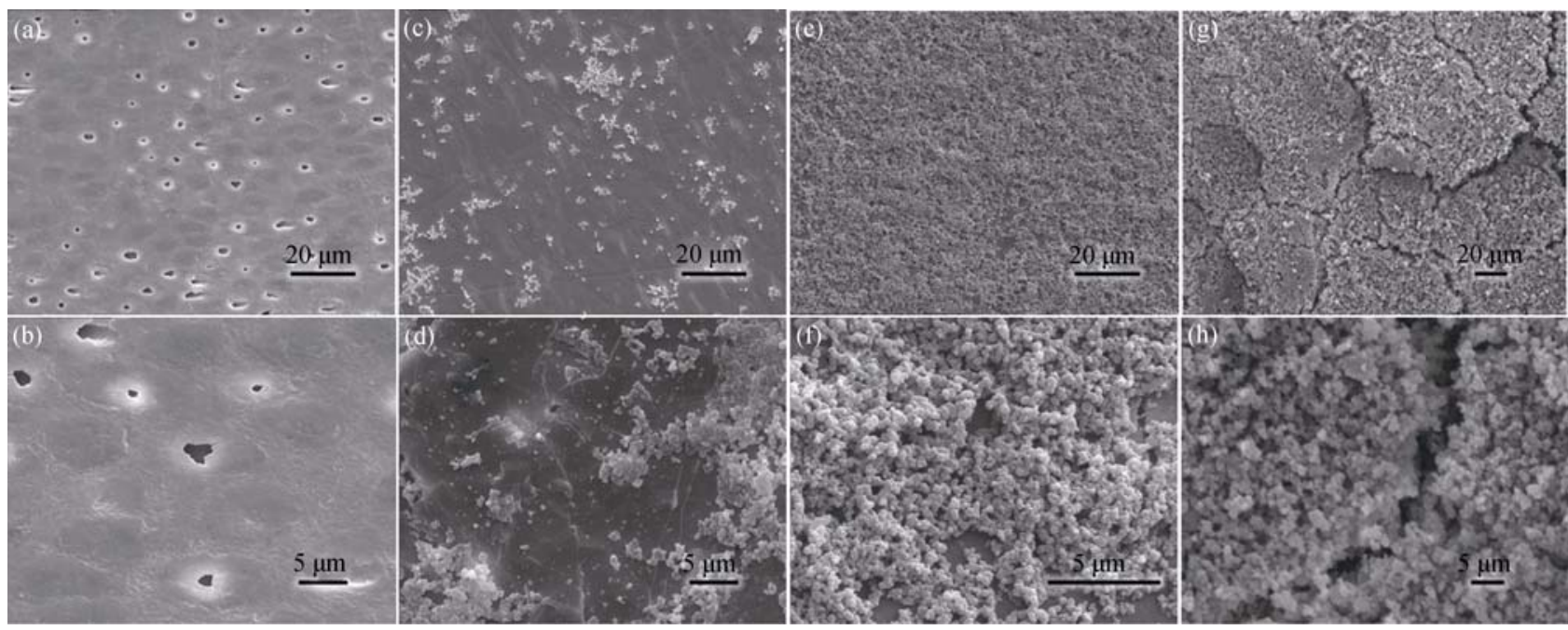

图 1 在不同浓度多巴胺溶液中处理后的牙本质表面 SEM 照片

Fig. 1 SEM images of the dentin surfaces after being soaked in dopamine solution at different concentrations, (a, b) $0 \mathrm{mg} / \mathrm{mL}$; (c, d) $1 \mathrm{mg} / \mathrm{mL}$; (e, f) $2 \mathrm{mg} / \mathrm{mL}$; (g, h) $3 \mathrm{mg} / \mathrm{mL}$ 
经低浓度多巴胺溶液处理后, 表面仅有少量聚多巴 胺(PDA)颗粒且分布不均匀(如图 1(c), (d)); 当将牙 片在 $2 \mathrm{mg} / \mathrm{mL}$ 的多巴胺溶液中处理 $24 \mathrm{~h}$ 并清洗、干 燥以后, 可观察到牙本质表面完全被 PDA 颗粒覆盖 (图 1(e), (f)), 且 PDA 层均匀致密; 当牙片经高浓度 多巴胺溶液处理后，牙本质表面沉积大量 PDA 颗粒， $\mathrm{PDA}$ 层较厚, 干燥之后 PDA 层出现裂纹, 局部出现 脱落。上述结果说明: $2 \mathrm{mg} / \mathrm{mL}$ 多巴胺溶液对牙本质 表面 PDA 层的形成最有效。

\section{2 多巴胺在牙本质表面聚合沉积状况的 XPS 结果分析}

对空白牙本质和沉积有聚多巴胺层的牙本质 (dentin-PDA)的 XPS 全谱图谱分析结果如图 2 所示, 比较 dentin-PDA(经 $2 \mathrm{mg} / \mathrm{mL}$ 多巴胺溶液处理后的 样品)与空白牙本质的谱图, 可以发现: 在空白牙本 质图谱中出现 P2p、P2s、Ca2s 峰, 而 dentin-PDA 谱图上这些峰极弱, 并且在 dentin-PDA 图谱中有与 牙本质的图谱中相同的 Ca2p、O1s、OKLL 峰, 但 峰强减弱, 这项结果说明所沉积的聚多巴胺层的厚 度小于 XPS 测试仪器对于有机基质探测的深度(约 为 $7.5 \mathrm{~nm})^{[15]}$; 在 dentin-PDA 图中 C1s 峰明显高于 空白牙本质, 这是由于沉积在牙本质表面的多巴胺 层中含有 $\mathrm{C}=\mathrm{C}$ 键、 $\mathrm{C}-\mathrm{C}$ 单键和 $\mathrm{C}-\mathrm{H}$ 键等, 说明在 空白牙本质表面有聚多巴胺的沉积 ${ }^{[16]}$ 。

在表 1 中所列的是由 XPS 测出的空白牙本质和 多巴胺改性过后的牙本质表面的化学元素组成。多 巴胺的分子理论 N/C 比值为 0.125 。XPS 测试所得 的空白牙本质表面元素的 N/C 比为 0.319 , 而经过 enamel-PDA 表面的 N/C 元素比为 0.179 , 介于空白 的牙本质和多巴胺的理论值之间，这也说明在空白 牙本质表面成功沉积有聚多巴胺涂层。

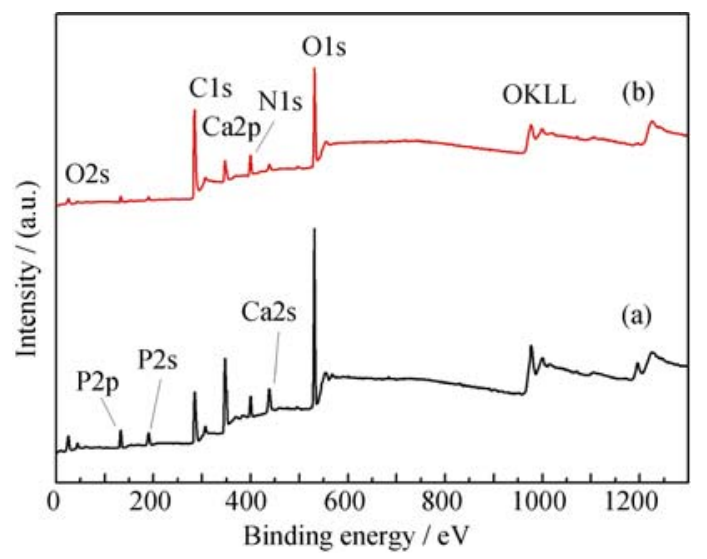

图 2 空白牙本质(a)和沉积有聚多巴胺层的牙本质(b)表面的 XPS 全谱图谱

Fig. 2 XPS wide scan spectra of $37 \% \mathrm{H}_{3} \mathrm{PO}_{4}$-etched dental slices (a) and the polydopamine coated dentin surface (b)
表 1 空白牙本质、多巴胺和表面沉积有多巴胺牙本质的表 面元素含量

Table 1 Element content of the dentin, dopamine and enamel-PDA surfaces

\begin{tabular}{cccc}
\hline & Dentin & $\begin{array}{c}\text { Dopamine } \\
\text { (theoretical value) }\end{array}$ & $\begin{array}{c}\text { Dentin } \\
\text {-PDA }\end{array}$ \\
\hline $\mathrm{C} 1 \mathrm{~s} / \mathrm{at} \%$ & 7.2 & 8 & 11.75 \\
$\mathrm{~N} 1 \mathrm{~s} / \mathrm{at} \%$ & 2.3 & 1 & 2.1 \\
$\mathrm{~N} / \mathrm{C}$ & 0.319 & 0.125 & 0.179 \\
\hline
\end{tabular}

\section{3 多巴胺诱导羟基磷灰石在牙本质表面沉积}

如图 3(c), 含聚多巴胺涂层的牙本质再矿化后 样本的 XRD 图谱显示, 衍射峰(002)、(211)、(112) 和(300)晶面都很好地对应着 HAP 的特征峰。含聚 多巴胺涂层的样本与酸蚀牙本质和无涂层的牙本质 相比, $c$-轴(002)衍射峰强度比(211)或 $a$-轴(300)的明显 增强，这表明 HAP 沉积物是沿着 $c$-轴生长的。图 3(b) 为无聚多巴胺涂层的牙本质上矿化沉积物样本的 XRD 图谱, $2 \theta$ 值为 $32^{\circ}$ 左右没有䢃裂的宽峰说明其 表面沉积的羟基磷灰石样品的结晶性比较差; 对比 图 3(b)与(c)可以看出, PDA 层有利于诱导牙本质表 面沿着 $c$-轴生长出羟基磷灰石晶体。这得益于聚多 巴胺涂层中的儿茶酚等基团与钙离子的结合, 使得 钙离子在表面富集而促进 HAP 的矿化 ${ }^{[17]}$ ，同时还 能调控 HAP 的结晶取向。

\section{4 多巴胺溶液诱导羟基磷灰石在牙本质表 面沉积的 SEM 形貌分析}

图 4(a)、(c)及其对应放大图(b)、(d)分别为涂覆有 PDA 涂层和无涂层的牙本质样本浸于再矿化液 $7 \mathrm{~d}$

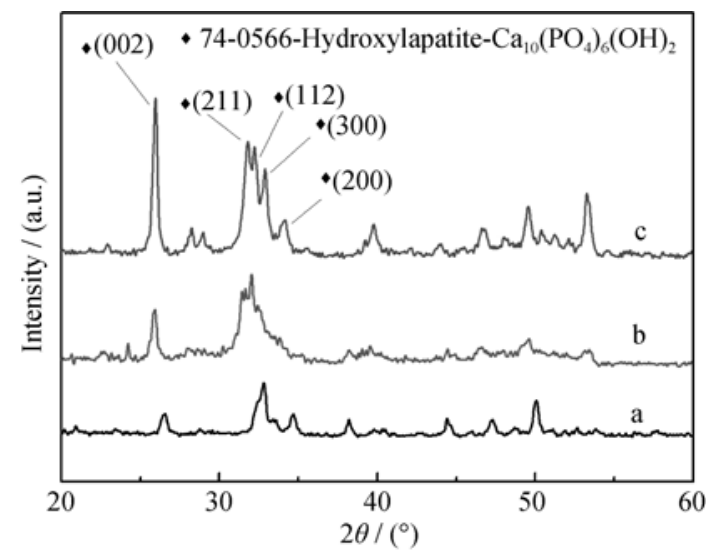

图 3 不同方式处理后的牙本质的 XRD 谱图: (a)空白牙本质; (b)无聚多巴胺涂层的牙本质再矿化; (c)含聚多巴胺涂层的牙 本质再矿化

Fig. 3 XRD patterns of the sample surfaces of $37 \% \mathrm{H}_{3} \mathrm{PO}_{4-}$ etched dental slices (a) and precipitates on the dental surface without (b) or with (c) polydopamine coating after remineralization for $7 \mathrm{~d}$ 
后表面 SEM 照片。从图中可以看出, 涂覆有 PDA 涂层的样本表面沉积物呈典型的天然鱼鳞结构, 沉 积物均匀排列且垂直于牙釉质表面定向结晶生长 (图 4(b)), 相互团簇形成“花朵”状且分布较均匀, 这 是由于这些 HAP 颗粒在牙本质上是沿着釉柱的方 向排列, 与牙本质本身的结构相似(图 4(a, b) $)^{[18] \text {; }}$ 无 PDA 涂层的样本表面沉积物呈短“线毛”结构, 局 部团聚成大颗粒散落在牙片表面, 这是由于矿化液 中产生的针状 HAP 晶体随机沉积而形成(图 4(c, d))。与无聚多巴胺涂层的样本相比, 聚多巴胺涂层 样本上的沉积晶体簇更趋于规则排列, 致密地聚集 成大的晶体。表 2 为含 PDA 涂层的样本再矿化后和 无 PDA 涂层的样本再矿化后表面微区 EDS 结果。 可以看出：含 PDA 涂层的样本各元素原子百分比与 标准羟基磷灰石原子百分比接近, 而无 PDA 涂层的 样本 $\mathrm{Ca} 、 \mathrm{P}$ 元素含量高于标准羟基磷灰石, 这是由 于其表面沉积了 $\mathrm{Ca} 、 \mathrm{P}$ 。

这些样本的断面 SEM 照片如图 5 所示, 对比原
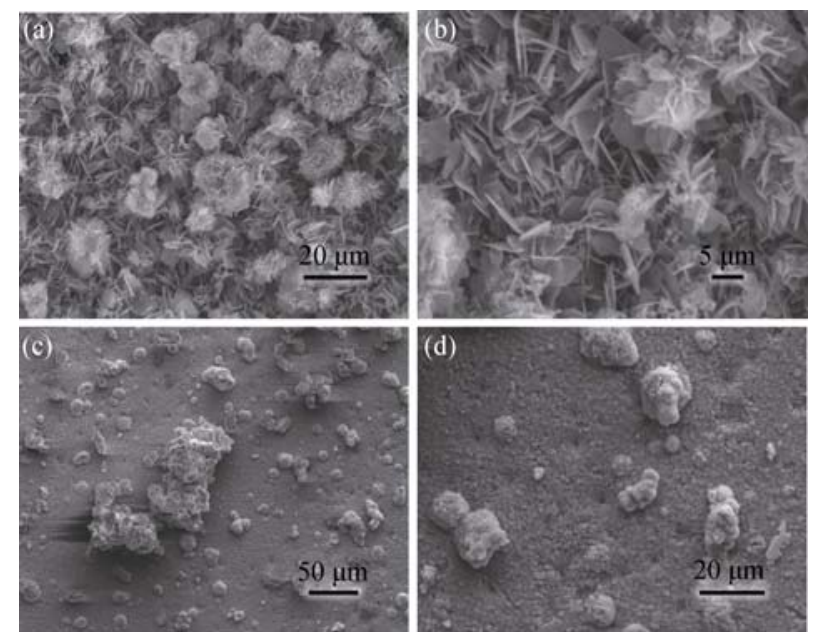

图 4 不同方式处理后的牙本质表面再矿化的 SEM 照片

Fig. 4 SEM images of HAP remineralization of the dentin surface coated with $(\mathrm{a}, \mathrm{b})$ or without $(\mathrm{c}, \mathrm{d})$ polydopamine after mineralization for $7 \mathrm{~d}$
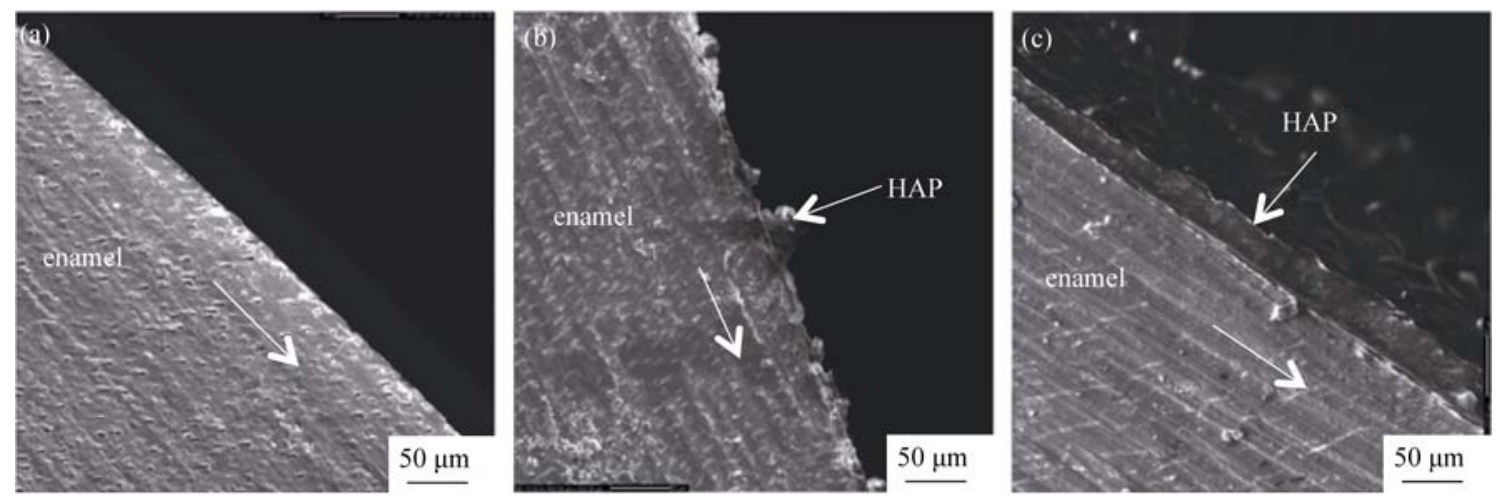

图 5 不同方式处理后的牙片断面 SEM 照片

Fig. 5 SEM images of the transverse sections of $37 \% \mathrm{H}_{3} \mathrm{PO}_{4}$-etched dental slices (a) and the preciptates on the enamel surface without (b) or with (c) polydopamine coating after remineralization for $7 \mathrm{~d}$ 

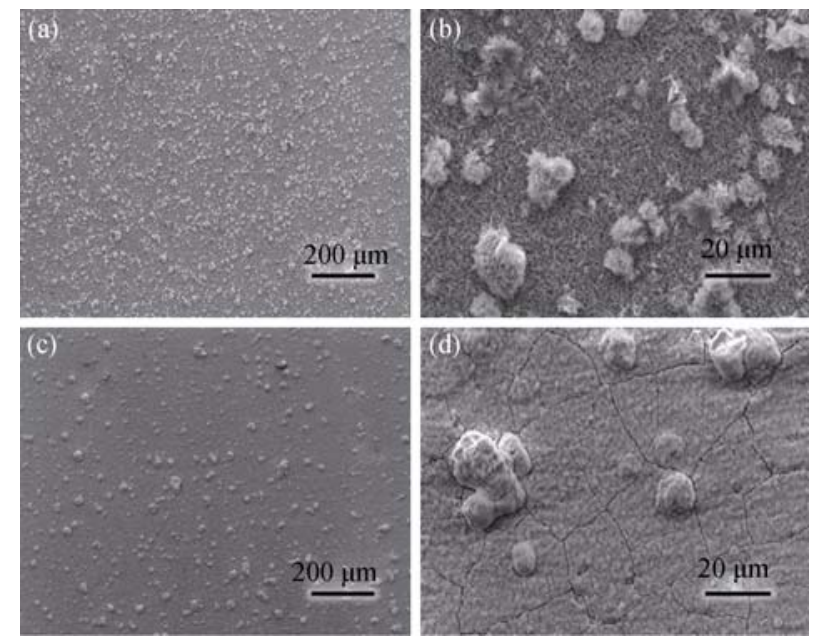

图 6 不同方式处理后的牙本质表面再矿化并超声后的 SEM 照片

Fig. 6 SEM images of dentin surface treated by ultrasonic wave which were remineralized with $(a, b)$ or without $(c, d)$ polydopamine coating after mineralization for $7 \mathrm{~d}$

\section{3 结论}

利用多巴胺自聚合使牙本质表面功能化的实验中, 制备 PDA 层的最佳多巴胺溶液浓度为 $2 \mathrm{mg} / \mathrm{mL}$ 。PDA 层可诱导牙本质表面在亚稳定的钻磷溶液中沿着 $c$-轴 生长出羟基磷灰石晶体, 与无 PDA 层的牙本质表面再 矿化效果相比, 涂覆有 PDA 层的牙本质表面再矿化层 均匀致密, 且与牙本质的结合力增强。该方法为具有良 好生物相容性的牙齿修复提供了新思路。

\section{参考文献:}

[1] FU HUA, YE TINFGTING, LV PEILIAN, et al. Introduction of clinical preventive medicine. Shanghai Journal of Preventive Medicine, 1998, (12): 551-553.

[2] LUO YING, CHENZHIQING, LIUZHAOHUI, et al. Effect of nano hydroxyapatite on demineralization of enamel. Journal of Sichuan University, 2002, 39(2): 286-289.

[3] MORADIAN-OLDAK J. Self-assembly properties of recombinant engineered amelogenin proteins analyzed by dynamic light scattering and atomic force microscopy. J. Struct. Biol., 2000, 131(1): 27-37.

[4] VEIS A. A window on biomineralization. Science, 2005, 307(5714): 1419-1420.

[5] JONES F H. Teeth and bones: applications of surface science to dental materials and related biomaterials. Surface Science Reports, 2011, 42(3): 75-205.
[6] GALLER K M, D'SOUZA R N, HARTGERINK J D. Biomaterials and their potential applications for dental tissue engineering, $J$. Mater. Chem., 2010, 20(40): 8730-8746.

[7] YAMAGISHI K, ONUMA K, SUZUKI T, et al. Materials chemistry: a synthetic enamel for rapid tooth repair. Nature, 2005, 433(7028): 819.

[8] CHEN H, CLARKSON B H, SUN K, et al. Self-assembly of synthetic hydroxyapatite nanorods into an enamel prism-like structure. Journal of Colloid \& Interface Science, 2005, 288(1): 97-103.

[9] CHEN H F, TANG Z Y, LIU J, et al. Acellular synthesis of a human enamel-like microstructure. Advanced Materials, 2006, 18(14): $1846-1851$.

[10] HONG X, XUE S, HUI M, et al. The preparation and antibacterial effects of dopa-cotton/AgNPs. Applied Surface Science, 2011, 257(15): 6799-6803.

[11] CUI J, WANG Y, POSTMA A, et al. Monodisperse polymer capsules: tailoring size, shell thickness, and hydrophobic cargo loading via emulsion templating. Advanced Functional Materials, 2010, 20(10): 1625-1631.

[12] YU B, WANG D A, YE Q, et al. Robust polydopamine nano/microcapsules and their loading and release behavior. Chemical Communications, 2009, 44(44): 6789-6791.

[13] YU F, CHEN S, CHEN Y, et al. Experimental and the oretical analysis of polymerization reaction process on the polydopamine membranes and its corrosion protection properties for 304 stainless steel. Journal of Molecular Structure, 2010, 982(1/2/3): 152-161.

[14] ZHANG H, YANG J, LIANG K, et al. Effective dentin restorative material based on phosphate-terminated dendrimer as artificial protein. Colloids Surf B: Biointerfaces, 2015, 128: 304-314.

[15] VORA R H, WANG W C, KANG E T, et al. Electroless plating of copper on fluorinated polyimide films modified by surface graft copolymerization with 1-vinylimidazole and 4-vinylpyridine. Polymer Engineering and Science, 2004, 44(2): 362-375.

[16] WU D, ZHANG T, WANG W C, et al. Reflective and conducitve surface-silvered polyimide films prepared by surface graft copolymerization and electroless plating. Polymers for Advanced Technologies, 2007, 19(5): 335-341.

[17] LIAO Y, CAO B, WANG W C, et al. A facile method for preparing highly conductive and reflective surface- silvered polyimide films. Applied Surface Science, 2009, 255(19): 8207-8212.

[18] BERNSMANN F, FRISCH B, RINGWALD C, et al. Protein adsorption on dopamine-melanin films: role of electrostatic interactions inferred from zeta-potential measurements versus chemisorption. Journal of Colloid \& Interface Science, 2010, 344(1): 54-60. 